\section{Association of anti-neutrophil cytoplasmic antibody in ischemic stroke Egyptian patients with hepatitis $\mathrm{C}$ virus}

\author{
Al-Amir B Mohamed ${ }^{1}$, Hesham M Hefny ${ }^{2}$, Mahmoud \\ Saif-Al-Islam ${ }^{3}$, Amr M Zaghloul ${ }^{3}$, Safaa Khalaf ${ }^{3}$, Ahmed \\ B Hassan ${ }^{1}$, Esam M Abualfadl ${ }^{4}$, Amal H Ali $^{5}$, and Hanan \\ SM Abozaid ${ }^{4}$
}

\author{
${ }^{1}$ Departments of Neurology, Faculty of Medicine, Sohag \\ University, Egypt. \\ ${ }^{2}$ Clinical Pathology, Faculty of Medicine, Sohag University, Egypt. \\ ${ }^{3}$ Tropical Medicine \& Gastroenterology, Faculty of Medicine, \\ Sohag University, Egypt. \\ ${ }^{4}$ Rheumatology \& Rehabilitation Department, Faculty of Medicine, \\ Sohag University, Egypt.
}

The Egyptian Journal of Immunology Volume 28 (1), 2021: 33-45. www.Ejimmunology.org

\begin{abstract}
Some studies reported a high prevalence of ischemic stroke in hepatitis $C$ virus patients, other several studies have suggested that hepatitis $\mathrm{C}$ virus (HCV) may act as a trigger for autoimmune diseases and autoantibodies including Anti-Neutrophil Cytoplasmic Antibody (ANCA) which predispose to vasculitis. Because vasculitis is a risk factor for ischemic stroke, we investigated the association of the hepatitis C virus with ANCA in first-ever ischemic stroke patients. This study included 67 Egyptian patients with first-ever ischemic stroke. These patients were clinically examined and investigated for HCV infection by chemiluminescence \& Real Time-PCR, and ANCA antibodies by ELISA. Forty-two patients (62.7\%) had HCV infection. Twenty-nine (43.2\%) of them were cytoplasmicAntineutrophil Cytoplasmic Antibodies (c-ANCA) positive, while none was perinuclear- Antineutrophil Cytoplasmic Antibodies ( $p$-ANCA) positive. Comparison between c-ANCA positive and ANCA negative patients showed that $82.8 \%$ and $47.4 \%$ had anti-HCV antibody, respectively, with $\mathrm{P}$-value 0.003 . The c-ANCA level correlated significantly with age, and HCV antibody level. No statistically significant difference was found in both the consciousness and stroke severity between the negative and positive c- ANCA patients. However, patients with positive c-ANCA had smaller and multiple cerebral infarctions with $P$-value 0.002 and 0.01 respectively. Multiple regression analysis showed that the number and size of cerebral infarctions were independent predictors of c-ANCA positivity with $P$ value 0.02 , and 0.03 respectively. In conclusion, c-ANCA level correlates with HCV antibody and may predispose to ischemic stroke by a possible ANCA associated vasculitis.
\end{abstract}

Keywords: Anti-Neutrophil Cytoplasmic Antibody (ANCA), Cerebro- vascular stroke (CVS), hepatitis C

Date received: 23 January 2021; accepted: 22 April 2021

\section{Introduction}

Stroke is the second leading cause of death worldwide and the main determinant of long- term disability. ${ }^{30}$ Infection appears to be an important trigger that precedes up to a third of ischemic strokes as inflammatory responses, which occur as a defense mechanism against 
infection can be a pathogenic mechanism that precipitates stroke and neurological sequelae. ${ }^{17}$

The extrahepatic manifestations can sometimes be the initial presentation of chronic HCV infection. Some of the extrahepatic manifestations have clear associations with the $\mathrm{HCV}$ infection, others were induced by immune responses triggered by HCV infection. The most common auto-immune diseases associated with chronic HCV infection are mixed cryoglobulinemia, autoimmune thyroiditis, nephropathy, sicca syndrome, idiopathic pulmonary fibrosis, lichen planus, type II diabetes mellitus, chronic polyarthritis, and porphyria cutanea tarda has been shown. ${ }^{26,48 \text {, }}$ 49,67

Autoimmune manifestations in chronic HCV infection are due to cross reactive immune reactions between epitopes detected on the viral polyprotein and human proteins inducing auto-antibodies causing tissue injury and inflammation. ${ }^{35,55,56,60}$

A population based cohort study concluded that chronic hepatitis $\mathrm{c}$ virus infection increases the risk of stroke and could be an independent risk factor for ischemic stroke. ${ }^{38}$ A recent metaanalysis reported a significantly increased risk of stroke in association with HCV infection ${ }^{27}$.

Cojocaru and colleagues reported that ANCA with anti- proteinase 3 (anti-PR3) specificity resulting from $\mathrm{HCV}$ infection were common, the authors also found that HCV patients with ANCA had a higher prevalence of ischemic stroke. ${ }^{13}$ Other reports did not found a significant association between HCV infection and stroke ${ }^{61,}$ 64 and this may be explained by the heterogeneity of the studied populations as regard gender, race, and hypertension the difference in the laboratory measurement into which the HCV was diagnosed only by anti HCV antibody and not using HCV RNA level.

The mechanism by which HCV may predispose to ischemic stroke is unclear. Atherosclerosis and carotid plaque destabilization with subsequent rupture and erosion plays a crucial role in the development of $20 \%-30 \%$ of all cases of ischemic stroke ${ }^{3}$. Inflammation is the key mediator of plaque rupture and thromboembolism. ${ }^{53}$ Occlusive cerebral vascular diseases can also occur in the context of HCVrelated vasculitis, such as mixed cryoglobulinemia, antiphospholipid syndrome, and anti-neutrophil cytoplasmic antibodies (ANCA)-associated vasculitis. ${ }^{51}$

The antineutrophil cytoplasmic antibody (ANCA) is a class of immunoglobulins features the classic cytoplasmic (c-ANCA) and the perinuclear ( $p$-ANCA) subtypes, which are predominantly generated against the cytosolic antigens proteinase 3 (PR3) and myeloperoxidase (MPO), respectively. ${ }^{29}$

Abnormal production of ANCAs can lead to neutrophil-mediated vascular injury in ANCAassociated vasculitis (AAV) which is diagnosed by biopsy. ${ }^{24}$ However, in clinical practice a biopsy is not always done, and a diagnosis is frequently made through clinical features with multiple organ affection including lung and kidneys involvement in combination with positive ANCA serology. Importantly, positive ANCA serology can also be found in other conditions with systemic symptoms, or even in asymptomatic patients. ${ }^{16,41}$

In this study we investigated the association between ANCA antibodies and HCV in patients with first ever ischemic stroke and find the relations between them and the neurological deficits.

\section{Patients and Methods}

This is a cross sectional study that included 67 patients who were admitted to Neurology department, Sohag University Hospital between December 2017 to December 2019. Only patients with first ever ischemic stroke were enrolled in this study.

Stroke, diagnosed with focal neurological signs or symptoms thought to be of vascular origin that persisted for $>24$ hours $^{33}$, confirmed by brain computed tomography (CT) and /or MRI. Those with clinical data corresponding to stroke with normal brain CT-Scan result was also considered as ischemic stroke but another CT scan was done after 2 days to measure the maximum lesion diameter.

The cerebral lesions whose maximum diameter was $\geq 4 \mathrm{~cm}$ were considered large infarction. ${ }^{43}$

Exclusion criteria included: Acute transient ischemic attack (TIA), recurrent stroke; intracerebral hemorrhage (ICH), decompensated liver disease, Hepatitis $C$ and $B$ coinfection and autoimmune diseases.

Patients with history of drug intake that may cause false positive ANCA test were also 
excluded. These drugs include Hydralazine, antithyroid drugs like propylthiouracil (PTU), minocycline, recreational drugs (cocaine and amphetamine), ergotamine and allopurinol.

Stroke severity was measured by the Scandinavian Stroke Scale (SSS) and categorized according to Stroke Unit Trialists' Collaboration into mild (43-58); moderate (26-42) and severe $(0-25) .^{58}$

Glasgow coma scale was used to assess conscious level of the patient and was graded as mildly disturbed (GCS=13-15), moderately disturbed (GCS $=9-12$ ), severely disturbed (GCS $\leq 8) .{ }^{57}$

The stroke outcome (within 1 month of admission) was determined using the modified Rankin Scale (mRS) which is graded from 0 to 6 points $^{9}$ applied within the first twenty-fourhours of admission (baseline). Patients with $\mathrm{mRS}<3$ was labeled as favorable outcome or independence (no symptoms/mild disability) and those with $\mathrm{mRS} \geq 3$ were labeled as unfavorable outcome or dependence (moderate/severe disability). ${ }^{54,59}$

All patients were managed using a standardized protocol adapted from international guidelines for managing acute ischemic stroke and none of them received thrombolytic therapy.

Demographic and clinical data and recorded traditional stroke risk factors before the inclusion in the study were obtained.

Each patient was subjected to the following: full medical examination including symptoms of upper airway obstruction, GIT and asthma. Also, symptoms of joint affection or skin rash are evaluated.

Full neurological evaluation including age, sex, traditional stroke risk factors and history of exposure to previous operations or blood transfusion with assessment of hepatic function including manifestations of hepatitis and liver cell failure such as jaundice, hepatomegaly, splenomegaly, ascites, and lower limb edema.

Assessment of blood pressure; high blood pressure was defined as; use of antihypertensive drugs or persistently elevated blood pressure ( $>140 / 90 \mathrm{mmHg}$ ) on admission.

Diabetes mellitus was defined as; use of hypoglycemic agents or a fasting plasma glucose of $>126 \mathrm{mg} / \mathrm{dl}$ (after no caloric intake for at least 8 hours) or, casual plasma glucose $>200$ $\mathrm{mg} / \mathrm{dl}$.

Abdominal Ultrasonography (U/S): Patients are subjected to $\mathrm{U} / \mathrm{S}$ examination to detect hepatic enlargement, focal lesion(s) and cirrhosis.

Laboratory investigations: $10 \mathrm{ml}$ of venous blood were withdrawn from each subject under aseptic conditions and by using sterile disposable gloves. The following investigations were performed:

1. Complete Blood Count (CBC): $\mathrm{CBC}$ was done by CEII - DYN 3700 (Abbott Laboratories, Diagnostic Division, IL, USA).

2. Biochemical assays for blood Glucose, Creatinine and Liver function tests including Alanine Aminotransferase (ALT), Aspartate Aminotransferase (AST), Alkaline phosphatase (ALP), Total bilirubin (TB) and Albumin were determined by Cobas c311 Chemistry Analyzer System (Roche Diagnostics $\mathrm{GmbH}$, Indianapolis, IN, USA).

3. Serological testing for both anti-HCV and HBsAg were done by Architect i1000SR system (Abbott Laboratories, Diagnostics Division, Abbott Park, IL).

4. Quantitative Real time PCR for the detection of HCV RNA and viral load:

A- Extraction) of RNA from serum: HCV viral RNA was extracted form serum samples by the use of fully automated QIAcube instrument and using specific kits; QIAgen columns (QIAamp Viral RNA Mini kit plus QIAmp Viral RNA Mini Accessory SET, cat. No. 1048147 Qlagen Inc), according to the manufacturer' instructions.

B- Real time quantitative PCR: HCV viral load was quantified using specific $\operatorname{TaqMAn}^{\circledR}$ probebased technology (QIAamp Viral RNA Mini Kit cat. No. 52904 Qiagen Inc) and 7500 Fast Real Time-PCR system (Applied Biosystems, CA, USA), according to the manufacturer' instructions. The reaction mixture was used in a total volume of $25 \mu$ including $10 \mu$ of the sample extract and $15 \mu$ of the Master Mix which prepared by: $6 \mu$ Hep. $C$ virus Master A and $9 \mu$ Hep. $C$ virus Master B. The real-time cycler conditions were performed according to the following conditions: Incubation at $50^{\circ} \mathrm{C}$ for $30 \mathrm{~min}$. then at $95^{\circ} \mathrm{C}$ for $15 \mathrm{~min}$. to activate the Taq enzyme followed by 50 amplification cycles. Each cycle consists of denaturation at $95^{\circ} \mathrm{C}$ for 
$30 \mathrm{sec}$, primer annealing at $50^{\circ} \mathrm{C}$ for $60 \mathrm{sec}$ and primer extension at $72^{\circ} \mathrm{C}$ for $30 \mathrm{sec}$.

5. Detection of Anti-PR3 (C-ANCA) and AntiMPO (P-ANCA) in serum:

Blood samples were withdrawn, left to clot at room temperature then centrifuged for 15 minutes at $1000 \times \mathrm{g}$. Serum was collected, divided into aliquots and stored at $-20^{\circ} \mathrm{C}$.

Both of Anti-PR3 and Anti-MPO were measured in serum samples using an enzymelinked immunosorbent assay (ELISA) kit (ORGENTEC Diagnostika, GmbH Carl-ZeissStraße 49-51, 55129 Mainz - Germany), according to the manufacturer' instructions. Patient samples were diluted 1:100 before the assay, $10 \mu \mathrm{l}$ of each sample was added to $990 \mu \mathrm{l}$ of prediluted sample buffer in a polystyrene tube and mixed well. Calibrators and controls are ready to use and need not be diluted. Calibrators, controls and prediluted patient samples were pipetted into the wells and incubated for 30 minutes at room temperature. Then, the contents of the microwells were discarded and washed 3 times. Horseradish peroxidase (HRP) conjugated anti-human antibodies was added to each well. Then, the plate was incubated for 15 minutes at room temperature. The contents of the microwells were discarded and washed 3 times. TMB substrate solution was added to each well and incubated for 15 minutes at room temperature. An enzyme substrate in the presence of HRP was catalyzed to form a blue colour. The reaction was stopped by addition of an acid stop solution forming a yellow color. The absorbance O.D. was measured at $450 \mathrm{~nm}$ using Thermo Fisher Scientific Multiskan EX Microplate Reader (Thermo Fisher Scientific Oy, FI-01621 Vantaa, Finland). The Anti-PR3 or AntiMPO concentration of samples, was determined by comparing the O.D. of the samples to a standard curve. The normal cut off healthy persons was less than $5 \mathrm{U} / \mathrm{ml}$.

lipogram was done and Cholesterol level was considered normal when total cholesterol is less than $200 \mathrm{mg} / \mathrm{dL}$, and LDL level is less than 100 $\mathrm{mg} / \mathrm{dL}$, while HDL level is $60 \mathrm{mg} / \mathrm{dL}$ or higher and normal Triglycerides is less than 150 $\mathrm{mg} / \mathrm{dL}^{18}$

\section{Statistical analysis}

The statistical analysis was performed using the Statistical Package for the Social Sciences for window (SPSS 20.0, IBM Corp., Armonk, NY, USA). All the patients' data were tabulated. Descriptive statistics were employed to investigate general characteristics of the patients. The patients were classified into 2 groups according to the presence or absence of c-ANCA. Continuous data were expressed as mean \pm SD, and categorical data were expressed as numbers and percentages. Comparisons of differences between two groups were performed by the Student's t-test for the continuous data, Chi-square test for categorical data. Association between c-ANCA levels and different categories $\mathrm{HCV}$ antibodies was determined by Pearson's product correlation and preliminary analyses were performed to ensure no violation of the assumptions of normality, linearity and homoscedasticity. All the predictive variables were first examined by logistic regression analysis. The analysis was performed by "enter method" among selection methods of variables. Odds ratio (OR) and $95 \%$ confidence interval $(95 \% \mathrm{Cl})$ were also determined. Survival curves were obtained using Kaplan-Meier's method. $P$ values of less than 0.05 were considered significant.

\section{Results}

The study included 67 patients with first ever ischemic stroke. Forty-two patients (62.7\%) were HCV antibodies positive and 25 patients (37.3\%) were HCV antibodies negative. The study included 36 males (53.7\%) and 31 female (46.2\%) patients. Sixteen patients $(23.8 \%)$ were smoker. Hypertension and DM were present in $(67 \%)$ and $(35.7 \%)$ respectively, $43.2 \%$ were cANCA positive Table (1).

For ANCA test, titers > 1: 40 were considered positive. All sera with ANCA showed c-ANCA pattern. None of the sera from ischemic stroke patients and HCV infection had p-ANCA. Table (1)

Most of the patients (67.1\%) had mildly disturbed conscious level by GCS and $52.2 \%$ of the patients have unfavorable outcome while death was reported in 12 patients (17.9\%), Table (1). 
Table 1. Clinical and laboratory characteristics of ischemic stroke Egyptian patients with hepatitis C virus.

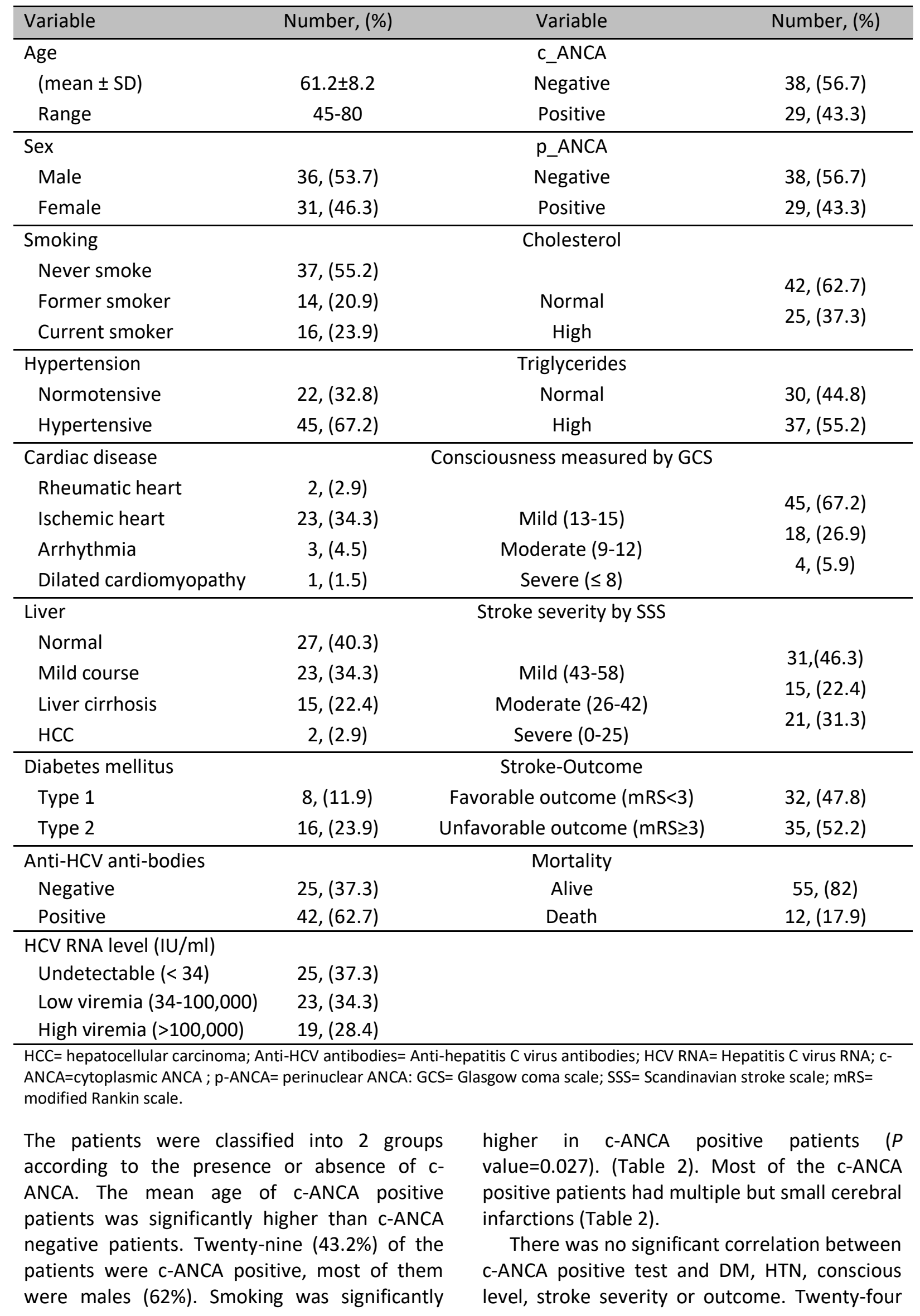


patients $(82.8 \%)$ of c-ANCA positive patients were anti-HCV antibody positive. Anti-HCV antibodies, high viremia and creatinine level, were significantly higher in c-ANCA positive patients, (Table 2). There were insignificant differences between c-ANCA negative and cANCA positive patients regarding level of serum cholesterol, triglycerides, glucose, prothrombin time and concentration, albumin, and bilirubin. Erythrocyte sedimentation rate (ESR) was slightly higher (although insignificantly) in cANCA positive patients (Table 2).

As for the survival rate, there was no significant difference between ANCA-positive and ANCA-negative stroke patients $(P=0.4$, Figure 1).

The mean age of patients with HCV antibody positive was higher the those with negative HCV but still insignificant $(P=0.05)$
More than $50 \%$ of patients with positive $\mathrm{HCV}$ had multiple cerebral infarctions with $P=0.01$. Table (3)

There was no significant correlation between positive HCV and conscious level stroke severity or outcome.

The relationship between C-ANCA and HCV infection (as measured by HCV antibodies) was investigated using Pearson product correlation coefficient. There was a Medium, positive correlation between the two variables [r.41, $\mathrm{n}=67, P=0.001]$. Table (4)

Multiple Logistic Regression Analyses of different variables with c-ANCA positive patients showed that the age, PCR level HCV viral RNA, number and size of cerebral infarctions were independent predictors of C-ANCA positivity with $P$ values $(0.028,0.018,0.02,0.037)$ respectively. (Table 5)

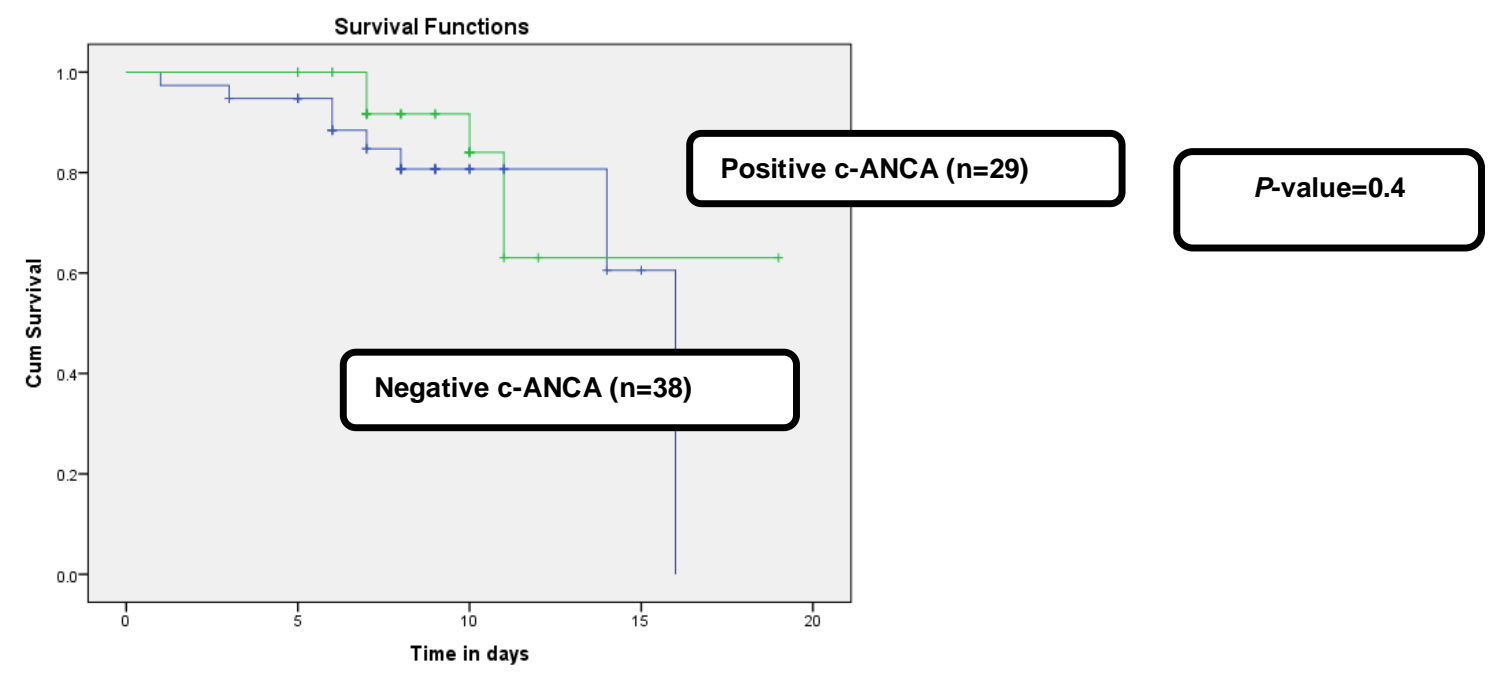

Figure 1. Kaplan-Meier's survival curves of ANCA-positive versus ANCA-negative ischemic stroke patients. As for the survival rate, there was no significant difference between ANCA-positive and ANCA-negative ischemic stroke Egyptian patients $(P=0.4)$

Table 2. c-ANCA negative and c-ANCA positive ischemic stroke Egyptian patients with Hepatitis C virus

\begin{tabular}{|c|c|c|c|c|}
\hline & & $\begin{array}{l}\text { Negative c- } \\
\text { ANCA }(N=38)\end{array}$ & Positive c-ANCA (N=29) & $P$-value \\
\hline Age & & $59.21 \pm 8.26$ & $63.97 \pm 7.60$ & 0.018 \\
\hline \multirow{2}{*}{ Sex: } & Male & 18 (47.37\%) & $18(62.07 \%)$ & \multirow{2}{*}{ NS } \\
\hline & Female & $20(52.63 \%)$ & 11 (37.93\%) & \\
\hline \multirow{3}{*}{ Smoking: } & Non-smoker & $26(68.42 \%)$ & $11(37.93 \%)$ & \multirow{3}{*}{0.027} \\
\hline & Former smoker & 7 (18.42\%) & 7 (24.14\%) & \\
\hline & Current smoker & 5 (13.16\%) & 11 (37.93\%) & \\
\hline \multicolumn{2}{|c|}{ Hypertension } & 25 (65.79\%) & 20 (68.97\%) & NS \\
\hline \multicolumn{2}{|c|}{ Cardiac disease } & $21(55.26 \%)$ & $8(27.59 \%)$ & 0.023 \\
\hline
\end{tabular}


Table 2. (Continued)

\begin{tabular}{|c|c|c|c|c|}
\hline & & $\begin{array}{l}\text { Negative c- } \\
\text { ANCA }(\mathrm{N}=38)\end{array}$ & $\begin{array}{c}\text { Positive c-ANCA } \\
(N=29)\end{array}$ & $\begin{array}{c}P- \\
\text { value }\end{array}$ \\
\hline \multirow{4}{*}{ Liver: } & Normal & $18(47.37 \%)$ & $9(31.03 \%)$ & \multirow{4}{*}{ NS } \\
\hline & Coarse echo pattern & $13(34.21 \%)$ & $10(34.48 \%)$ & \\
\hline & Liver cirrhosis & $6(15.79 \%)$ & 9 (31.03\%) & \\
\hline & $\mathrm{HCC}$ & $1(2.63 \%)$ & $1(3.45 \%)$ & \\
\hline \multirow{3}{*}{$\begin{array}{l}\text { Diabetes } \\
\text { mellitus: }\end{array}$} & Non-diabetic & 25 (65.79\%) & $18(62.07 \%)$ & \multirow{3}{*}{ NS } \\
\hline & Type 1 & $5(13.16 \%)$ & $3(10.35 \%)$ & \\
\hline & Type 2 & $8(21.05 \%)$ & $8(27.59 \%)$ & \\
\hline \multirow{3}{*}{ GCS: } & Mildly disturbed & $24(63.16 \%)$ & $21(72.41 \%)$ & \multirow{3}{*}{ NS } \\
\hline & Moderately disturbed & $10(26.32 \%)$ & $8(27.59 \%)$ & \\
\hline & Severely disturbed & $4(10.53 \%)$ & $0(0.0 \%)$ & \\
\hline \multirow{3}{*}{ SSS: } & Mild & $18(47.37 \%)$ & $13(44.83 \%)$ & \multirow{3}{*}{ NS } \\
\hline & Moderate & 7 (18.42\%) & $8(27.59 \%)$ & \\
\hline & Severe & $13(34.21 \%)$ & $8(27.59 \%)$ & \\
\hline \multirow{2}{*}{$\begin{array}{l}\text { Radiological data } \\
\text { (CT \&/or MRI): }\end{array}$} & Single lesion & $25(65.8 \%)$ & $10(34.5 \%)$ & \multirow[b]{2}{*}{0.011} \\
\hline & Multiple lesions & $13(34.2 \%)$ & $19(65.5 \%)$ & \\
\hline \multirow{2}{*}{ Lesion size $(\mathrm{cm})$ : } & Small Infarction $(<4 \mathrm{~cm})$ & $16(42.1 \%)$ & $23(79.3 \%)$ & 0.002 \\
\hline & Large Infarction $(\geq 4 \mathrm{~cm})$ & $22(57.9 \%)$ & $6(20.7 \%)$ & 0.002 \\
\hline \multirow{4}{*}{ Abdominal U/S: } & Hepatomegaly & $1(2.6 \%)$ & $2(6.9 \%)$ & NS \\
\hline & Mild course & $13(34.2 \%)$ & $10(34.5 \%)$ & \multirow[t]{3}{*}{ NS } \\
\hline & Liver cirrhosis & $6(15.8 \%)$ & $9(31.0 \%)$ & \\
\hline & $\mathrm{HCC}$ & $1(2.6 \%)$ & $1(3.4 \%)$ & \\
\hline \multirow{2}{*}{$\begin{array}{l}\text { Outcome } \\
\text { (measured } \\
\text { Mrs): }\end{array}$} & Favorable outcome & 19 (50\%\%) & 13 (44.8\%\%) & \multirow{2}{*}{ NS } \\
\hline & Unfavorable outcome & $19(50 \% \%)$ & $16(55.2 \% \%)$ & \\
\hline \multirow{2}{*}{ Mortality: } & Alive & $30(78.95 \%)$ & $25(86.21 \%)$ & \multirow[b]{2}{*}{ NS } \\
\hline & Death & $8(21.05 \%)$ & $4(13.79 \%)$ & \\
\hline \multicolumn{2}{|l|}{ Cholesterol (mg/dl) } & $186.42 \pm 54.55$ & $187.24 \pm 53.98$ & NS \\
\hline \multicolumn{2}{|c|}{ Triglycerides (mg/dl) } & $164.87 \pm 68.55$ & $203.66 \pm 126.73$ & NS \\
\hline \multicolumn{2}{|c|}{ Blood glucose (mg/dl) } & $150.16 \pm 58.92$ & $137.86 \pm 55.91$ & NS \\
\hline \multicolumn{2}{|c|}{ Serum creatinine $(\mathrm{mg} / \mathrm{dl})$} & $1.16 \pm 1.24$ & $2.15 \pm 1.9$ & 0.014 \\
\hline \multicolumn{2}{|c|}{ Prothrombin time (sec) } & $12.99 \pm 1.85$ & $13.02 \pm 1.41$ & NS \\
\hline \multicolumn{2}{|c|}{ Prothrombin concentration (\%) } & $83.53 \pm 14.35$ & $82.18 \pm 12.79$ & NS \\
\hline \multicolumn{2}{|l|}{ Albumin (gm/dl) } & $4.23 \pm 5.50$ & $3.28 \pm 0.72$ & NS \\
\hline \multicolumn{2}{|c|}{ Total bilirubin (mg/dl) } & $1.01 \pm 0.69$ & $1.03 \pm 0.74$ & NS \\
\hline \multicolumn{2}{|c|}{$\operatorname{RBCs}\left(\times 10^{12} / \mathrm{I}\right)$} & $4.66 \pm 0.98$ & $4.61 \pm 0.61$ & NS \\
\hline $\mathrm{ESR}(\mathrm{mm})$ & & $30.53 \pm 29.29$ & $36.48+30.00$ & NS \\
\hline Anti-HCV & Negative & $20(52.6 \%)$ & $5(17.2 \%)$ & \\
\hline antibodies: & Positive & $18(47.4 \%)$ & $24(82.8 \%)$ & 0.003 \\
\hline & Undetectable $(<34)$ & $20(52.6 \%)$ & $5(17.2 \%)$ & \\
\hline HCV RINA level & Low level (34-100000) & $12(31.6 \%)$ & $11(37.9 \%)$ & 0.005 \\
\hline$(1 \mathrm{U} / \mathrm{ml})$ & High level (>100000) & $6(15.8 \%)$ & $13(44.8 \%)$ & \\
\hline HCV RNA level by & PR (mean $\pm S D)$ & $167549.95+430737.6$ & $\begin{array}{c}700011.48+123871 \\
9.554\end{array}$ & 0.016 \\
\hline
\end{tabular}

HCC= Hepatocellular carcinoma. GCS= Glasgow coma scale. SSS= Scandinavian stroke scale. mRS= modified Rankin scale. $\mathrm{RBCS}=$ Red blood cells. ESR= Erythrocyte sedimentation rate. Anti-HCV antibodies= Anti-hepatitis C virus antibodies. HCV RNA $=$ Hepatitis $C$ virus RNA. HCV RNA= hepatitis $C$ virus RNA by polymerase chain reaction. $P>0.05$ is not significant (NS) 
Table 3. Clinical characteristics of HCV negative versus HCV positive ischemic stroke Egyptian patients.

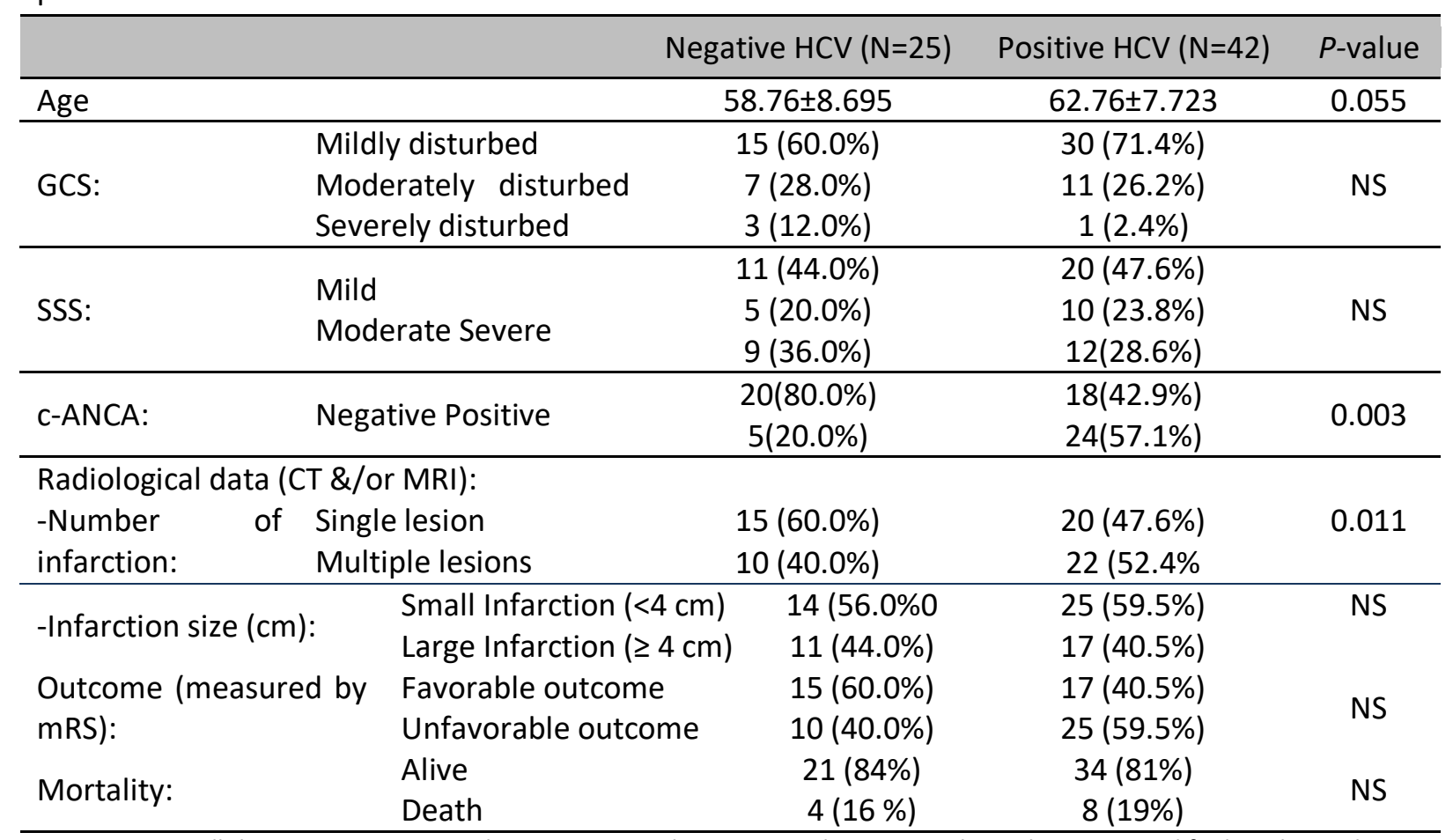

$\mathrm{HCC}=$ Hepatocellular carcinoma. GCS= Glasgow coma scale. SSS= Scandinavian stroke scale. mRS= modified Rankin scale $P>0.05$ is not significant (NS)

Table 4. Correlation between c-ANCA and HCV antibody in ischemic stroke patients.

\begin{tabular}{llcc}
\hline & & C-ANCA & HCV antibody \\
\hline \multirow{2}{*}{ C-ANCA } & Pearson Correlation & 1 & 0.412 \\
& Sig. (2-tailed) & & $* 0.001$ \\
& Number & 67 & 67 \\
\hline \multirow{3}{*}{ Serology } & Pearson Correlation & 0.412 & 1 \\
& Sig. (2-tailed) & $* 0.001$ & 67 \\
& Number & 67 &
\end{tabular}

${ }^{*} P<0.05$ is Significant.

Table 5. Multiple Logistic Regression Analyses of different variables in c- ANCA positive ischemic stroke patients.

\begin{tabular}{|c|c|c|c|c|c|c|}
\hline & \multirow{2}{*}{$\begin{array}{l}\text { Regression } \\
\text { Coefficient }\end{array}$} & \multirow{2}{*}{ Std. Error } & \multirow{2}{*}{$P$-value } & \multirow{2}{*}{$\begin{array}{l}\text { Odds } \\
\text { ratio }\end{array}$} & \multicolumn{2}{|c|}{ 95\% C.I. } \\
\hline & & & & & Lower & Upper \\
\hline Age & 0.129 & 0.059 & 0.028 & 1.138 & 1.014 & 1.277 \\
\hline HCV antibody & -0.193 & 0.996 & NS & 0.824 & 0.117 & 5.807 \\
\hline PCR level & 0.611 & 0.257 & 0.018 & 1.842 & 1.113 & 3.051 \\
\hline Number of cerebral infarctions & 1.899 & 0.819 & 0.020 & 6.680 & 1.341 & 33.272 \\
\hline Size of cerebral infarction & -1.636 & 0.783 & 0.037 & 0.195 & 0.042 & 0.904 \\
\hline
\end{tabular}

Age, PCR level the number and size of cerebral infarction were independent predictors of c-ANCA positivity.

$P>0.05$ is not significant (NS).

\section{Discussion}

Stroke is still a major global health problem in both developed and developing countries and determining the stroke subtype and underlying etiologies is a major step to choose the best method for prophylaxis. ${ }^{25}$

Chronic hepatitis C virus HCV may act as a trigger for the development of autoimmune diseases $^{22}$, one of these autoimmune diseases is 
autoimmune vasculitis. Vasculitis of the cerebral vessels can cause ischemic stroke. Ischemic stroke is one of the life-threatening complications of vasculitis in the central nervous system in chronic HCV infection. ${ }^{34}$ Therefore, the present study was conducted to clarify the different characteristics of ischemic stroke in negative and positive c-ANCA test and to investigate the possible occurrence of cerebral vasculitis as a cause of ischemic stroke in patients with HCV and positive c-ANCA test.

In the absence of biopsy, the ANCA titre and the number of affected organ systems could be considered as diagnostic markers of ANCA Associated Vasculitis (AAV) in clinical practice. ${ }^{31}$ In rare cases of AAV, the initial symptoms are those of cerebral infarction, which is difficult to distinguish from atherothrombotic cerebral infarction, leading to possible treatment delays. $^{63}$

In our study, the prevalence of HCV infection in patients with ischemic stroke was high (62.7\%). This reflects the high prevalence of HCV in our country in addition to the possible role of $\mathrm{HCV}$ in induction of ischemic stroke. Several authors documented the association between chronic

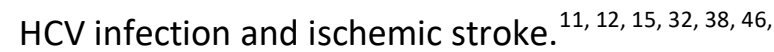
66

$\mathrm{HCV}$ infection is associated with an increased risk of cerebrovascular mortality, particularly for those with elevated serum HCV RNA levels. ${ }^{37}$ and, a recent meta-analysis concluded for a significantly increased risk of stroke in association with $\mathrm{HCV}$ infection. ${ }^{27}$

In contrast, Younossi et al. ${ }^{65}$ were not able to demonstrate an association between HCV and stroke in a retrospective cohort of subjects enrolled in the NHANES database between 1999 and 2010, the study population were heterogeneous in terms of such factors as gender, race, and hypertension.

The mechanism by which HCV may predispose to ischemic stroke is unclear, However, HCV induced vasculitis is a possible mechanism. Chronic HCV infection is characterized by a state of chronic inflammation, which may be fundamental in the pathogenesis of ischemic stroke ${ }^{65}$ In addition, lines of evidence suggest that the brain, but not peripheral nerves or skeletal muscles, is a permissive site for viral replication, as evinced from quasispecies analysis and the detection of replicative intermediate forms of HCV RNA and viral proteins within the central nervous system (CNS). ${ }^{19}$

Previous studies have shown the expression of HCV receptors in the brain microvascular endothelial cells and the microvascular endothelia are the only cells in the neuronal pool to bear the receptors for HCV. Microvascular endothelial cells, that form integral components of blood brain barrier (BBB), are thus assumed to play critical role in the transit of $\mathrm{HCV}$ into CNS. ${ }^{20,40}$

Additional mechanisms, contributing to neurological dysfunction, are possibly related to the effect of circulating inflammatory cytokines and chemokines reaching brain tissues across altered sites of the blood-brain barrier. ${ }^{42}$

Cryoglobulinemia, is detected in up to $50 \%$ of $\mathrm{HCV}$ infected patients, inducing symptomatic disease in nearly $15 \%$ of cases. Cryoglobulins (CGs) are cold-precipitable immunoglobulins, which, following vascular deposition, elicit inflammation and occlusion of small- and medium size blood vessels. ${ }^{42}$

Some studies have noted an association between carotid artery atherosclerosis and HCV infection. $^{6,23}$ and HCV RNA sequences have been detected in carotid artery plaque from patients seropositive for antibody to $\mathrm{HCV}^{7}$

Up to our knowledge this study is one of the few studies that assess the relation between cANCA and HCV in patients with ischemic stroke.

In our study we found that most of c-ANCA positive patients $(82.8 \%)$ were anti HCV antibodies positive indicating a significant association between chronic HCV infection and c-ANCA positivity.

ANCA induction can occur due to many chronic infections including chronic HCV infection. About thirty-five percent (35.2\%) of patients with positive ANCA had confirmed viral infections including $\mathrm{HCV}^{8}$ The high percent of positive ANCA in our series related to the usage of more sensitive ELIZA method for detection of ANCA and higher prevalence of HCV in our locality

Acay and colleagues reported that the frequency of auto-antibodies particularly antinuclear antibody (ANA) is significantly increased among individuals with HCV infection and it was detected in nearly $20 \%$ of $\mathrm{HCV}$ infected patients. ${ }^{2}$ 
Another study reported that approximately 20$40 \%$ of patients with chronic HCV hade nonorgan specific autoantibodies. Female gender and significantly more elevated ALT values are prevalent in these cases. ${ }^{10}$

In a study was conducted in 2 European centers, ANCA was present in about $10 \%$ of patients with HCV-associated mixed cryoglobulinemia or chronic hepatitis $C .{ }^{36}$

In this work c-ANCA positivity is significantly associated with smaller size and the larger number of cerebral infarctions which raise the possibility of vasculitis as a cause of cerebral infarction in HCV patients. Like previous studies that documented CNS vasculitis as well-known events in patients with HCV. ${ }^{5,15,28}$ Malnick and colleagues attributed the CNS ischemic changes to an anti-phospholipid syndrome or it might be associated with the anti-neutrophil cytoplasmic antibodies. $^{39}$

In our series the c-ANCA positivity not correlated to conscious level, stroke severity, outcome or mortality as most of the patients presented with lacunar infarcts, a finding confirmed by other studies which reported the occurrence of acute cerebrovascular events including transient ischemic attacks, ischemic stroke and lacunar syndromes in patients suffering with $\mathrm{HCV}$. $45,47,50$

The mean age of c-ANCA positive patients was significantly higher than c-ANCA negative patients denoting that c-ANCA positivity may be gradually acquired with increasing duration of HCV infection. Thus, vasculitis becomes more common with increasing age. c-ANCA positivity was more common in males which may indicate early acquisition of $\mathrm{HCV}$ infection in males than in females. Watts et al. (2012) ${ }^{62}$ found the peak age at diagnosis of ANCA positivity was 65-74 years with a male preponderance. Many authors found higher prevalence of $\mathrm{HCV}$ in males compared to females. ${ }^{4,53}$ with marked increase in prevalence with increase in age $\mathrm{e}^{1,14,45}$. The higher prevalence observed in males and rural dwellers may be in part attributed to the parenteral anti-schistosomiasis therapy campaigns. $^{21}$

Smoking were significantly higher in c-ANCA positive patients. Smoking leads to multiple system affection and inflammation. Chronic inflammation is linked to c-ANAC induction.
In our study there were insignificant differences between C-ANCA negative and C-ANCA positive patients regarding level of serum cholesterol, triglycerides, glucose, creatinine, prothrombin time and concentration, albumin, and bilirubin. Erythrocyte sedimentation rate (ESR) was slightly higher (although insignificantly) in ANCA positive patient.

Limitations: The major limitation of this study is relatively small number of patients and it is not designed prospectively, we did not follow the patients to detect the emergence of autoimmune diseases. Despite these limitations, the present study has shown that a significant association between HCV infection and ANCA. ANCA-associated vasculitis (AAV) may be considered as possible cause of stroke, so ANCA should be screened for patients with stroke and infected by HCV and should be treated by specific strategies in order to prevent cerebrovascular disease.

In conclusion, the present study is among the few studies that exhibit an association between ANCA and HCV infection in patients with first ever ischemic stroke and confirm that AAV could be a possible cause of stroke.

\section{Author Contributions}

$A B M$ and $A B H$, managed the research protocol, diagnosed, assessed the neurological lesions, and shared in the statistical work and writing the manuscript. $\mathrm{HMH}$, managed the research protocol, did the laboratory work, shared in writing the final manuscript. MS, AMZ, SK, managed the protocol, diagnosed, and assessed the hepatic condition, shared in writing. EMA, HSMA, managed the protocol of the study, clinically assessed the patients, writing the manuscript, statistical work. AHA, managed the protocol, shared in the laboratory work. All authors read and approved the final manuscript.

\section{Declaration of Conflicting Interests}

The author(s) declared no potential conflicts of interest with respect to the research, authorship, and/or publication of this article.

\section{Funding}

The authors received no financial support for the research, authorship, and/or publication of this article 


\section{Ethical approval}

The study protocol got ethical approval number IBR\# S20-137 from Sohag University, Faculty of Medicine, and research ethical committee.

\section{Informed consent}

A signed consent form was obtained from each study participant.

\section{References}

1. Fatma Abdel-Aziz, Mostafa Habib, Mostafa K. Mohamed, et al. (2000) Hepatitis C Virus(Hcv) Infection in a Community in the Nile Delta: Population Description and Hcv Prevalence', Hepatology, 32, 111-15.

2. Akif Acay, Kasim Demir, Gulsah Asik, et al. (2015) Assessment of the Frequency of Autoantibodies in Chronic Viral Hepatitis', Pakistan journal of medical sciences, 31, 150.

3. Luigi E. Adinolfi. (2014) Chronic Hepatitis C Virus Infection and Atherosclerosis: Clinical Impact and Mechanisms', World Journal of Gastroenterology, 20, 3410 .

4. Naglaa Arafa, Mostafa El Hoseiny, Claire Rekacewicz, et al. (2005) Changing Pattern of Hepatitis C Virus Spread in Rural Areas of Egypt', Journal of Hepatology, 43, 418-24.

5. MG Arena, E Ferlazzo, D Bonanno, $P$ et al. (2003) Cerebral Vasculitis in a Patient with Hcv-Related Type li Mixed Cryoglobulinemia', Journal of investigational allergology \& clinical immunology, 13, 135-36.

6. F. Aslam, M. Alam, and N. M. Lakkis. (2010) Hepatitis C and Carotid Atherosclerosis: A Retrospective Analysis', Atherosclerosis, 209, 340-3.

7. M. Boddi, R. Abbate, B. Chellini, B. et al. (2010) Hepatitis C Virus Rna Localization in Human Carotid Plaques', J Clin Virol, 47, 72-5.

8. Branka Bonaci-Nikolic, Sladjana Andrejevic, Milorad Pavlovic, et al. (2010) Prolonged Infections Associated with Antineutrophil Cytoplasmic Antibodies Specific to Proteinase 3 and Myeloperoxidase: Diagnostic and Therapeutic Challenge', Clinical Rheumatology, 29, 893-904.

9. R. Bonita, and R. Beaglehole. (2001) Recovery of Motor Function after Stroke', Stroke, 19, 1497-500.

10. Thomas V Cacciarelli, Jorge Reyes, Ronald Jaffe, et al. (2001) Primary Tacrolimus (Fk506) Therapy and the Long-Term Risk of Post-Transplant Lymphoproliferative Disease in Pediatric Liver Transplant Recipients', Pediatric transplantation, 5, 359-64.

11. P. Cacoub, A. Sbaï, P. Hausfater, et al. (1998) [Central Nervous System Involvement in Hepatitis C Virus Infection]', Gastroenterol Clin Biol, 22, 631-3.
12. Roberto J. Carvalho-Filho. (2012) Central Nervous System Vasculitis and Polyneuropathy as First Manifestations of Hepatitis C', World Journal of Gastroenterology, 18, 188.

13. I. M. Cojocaru, M. Cojocaru, and C. Burcin. (2007) Ischemic Stroke Accompanied by Anti-Pr3 AntibodyRelated Cerebral Vasculitis and Hepatitis C Virus Infection', Rom J Intern Med, 45, 47-50.

14. Medhat A. Darwish, Malla R. Rao, et al. (1996) High Seroprevalence of Hepatitis a, B, C, and E Viruses in Residents in an Egyptian Village in the Nile Delta: A Pilot Study', The American Journal of Tropical Medicine and Hygiene, 54, 554-58.

15. T. M. Dawson, and G. Starkebaum. (1999) Isolated Central Nervous System Vasculitis Associated with Hepatitis C Infection', J Rheumatol, 26, 2273-6.

16. M De Bandt, O Meyer, T Haim, et al. (1996) Antineutrophil Cytoplasmic Antibodies in Rheumatoid Arthritis Patients', Rheumatology, 35, 38-43.

17. Hedley CA Emsley, and Stephen J Hopkins. (2008) Acute Ischaemic Stroke and Infection: Recent and Emerging Concepts', The Lancet Neurology, 7, 34153.

18. Evaluation Expert Panel on Detection, 'Executive Summary of the Third Report of the National Cholesterol Education Program (Ncep) Expert Panel on Detection, Evaluation, and Treatment of High Blood Cholesterol in Adults (Adult Treatment Panel lii)', Jama, 285, 2486.

19. NF Fletcher, and JA McKeating. (2012) Hepatitis C Virus and the Brain, Journal of viral hepatitis, 19, 301-06.

20. Nicola F Fletcher, Jian Ping Yang, Michelle J Farquhar, et al. (2010)Hepatitis C Virus Infection of Neuroepithelioma Cell Lines', Gastroenterology, 139, 1365-74. e2.

21. Christina Frank, Mostafa K. Mohamed, G. Thomas Strickland, et al. (2000) The Role of Parenteral Antischistosomal Therapy in the Spread of Hepatitis C Virus in Egypt', The Lancet, 355, 887-91.

22. H. L. Fu, T. C. Hsu, C. C. Chang, et al. (2001) Antigenic Specificity of Anti-Neutrophil Cytoplasmic Antibody', J Formos Med Assoc, 100, 35-9.

23. M. Fukui. (2003) Hepatitis C Virus and Atherosclerosis in Patients with Type 2 Diabetes', JAMA: The Journal of the American Medical Association, 289, 1245-a-46.

24. Angelo L Gaffo. (2010) Diagnostic Approach to Anca-Associated Vasculitides', Rheumatic Disease Clinics, 36, 491-506.

25. Levent Gungor, Murat Polat, Mehlika Berra Ozberk, et al. (2018) Which Ischemic Stroke Subtype 
Is Associated with Hyperhomocysteinemia?', Journal of Stroke and Cerebrovascular Diseases, 27, 1921-29.

26. Joel Haddad, JC Trinchet, D Pateron, et al. (1992) Lymphocytic Sialadenitis of Sjögren's Syndrome Associated with Chronic Hepatitis C Virus Liver Disease', The Lancet, 339, 321-23.

27. He Huang, R. Kang, and Z. Zhao. (2013) Hepatitis $C$ Virus Infection and Risk of Stroke: A Systematic Review and Meta-Analysis', PLoS One, 8, e81305.

28. JG Heckmann, C Kayser, D Heuss, et al. (1999) Neurological Manifestations of Chronic Hepatitis C', Journal of neurology, 246, 486-91.

29. B. Hellmich, M. Ehren, M. Lindstaedt, et al. (2001) Anti-Mpo-Anca-Positive Microscopic Polyangiitis Following Subacute Bacterial Endocarditis', Clinical Rheumatology, 20, 441-43.

30. J. C. Hopewell, and R. Clarke. (2016) Emerging Risk Factors for Stroke: What Have We Learned from Mendelian Randomization Studies?', Stroke, 47, 1673-8.

31. Eline Houben, Willem A Bax, Bastiaan van Dam, et al. (2016) Diagnosing Anca-Associated Vasculitis in Anca Positive Patients: A Retrospective Analysis on the Role of Clinical Symptoms and the Anca Titre', Medicine, 95

32. C. S. Hsu, J. H. Kao, Y. C. Chao, et al. (2013) Interferon-Based Therapy Reduces Risk of Stroke in Chronic Hepatitis C Patients: A Population-Based Cohort Study in Taiwan', Aliment Pharmacol Ther, 38, 415-23.

33. Who Monica Project Principal Invest. The World Health Organization Monica Project (Monitoring Trends and Determinants in Cardiovascular Disease): A Major International Collaboration', Journal of Clinical Epidemiology, 41, 105-14.

34. J. Charles Jennette, and Ronald J. Falk. (1997) Small-Vessel Vasculitis', New England Journal of Medicine, 337, 1512-23.

35. John Koskinas, Barbara M. McFarlane, Kayhan T. Nouri-Aria, et al. (1994) Cellular and Humoral Immune Reactions against Autoantigens and Hepatitis C Viral Antigens in Chronic Hepatitis C', Gastroenterology, 107, 1436-42.

36. P Lamprecht, O Gutzeit, E Csernok, et al. (2003). Prevalence of Anca in Mixed Cryoglobulinemia and Chronic Hepatitis C Virus Infection', Clinical and experimental rheumatology, 21, S89-S94.

37. Mei-Hsuan Lee, Hwai-I Yang, Chih-Hao Wang, et al. (2010) Hepatitis C Virus Infection and Increased Risk of Cerebrovascular Disease', Stroke, 41, 2894900.

38. C. C. Liao, T. C. Su, F. C. Sung, et al. (2012) Does Hepatitis C Virus Infection Increase Risk for Stroke? A Population-Based Cohort Study', PLoS One, 7, e31527.
39. S. D. Malnick, Y. Abend, E. Evron, et al. (1997) 'Hcv Hepatitis Associated with Anticardiolipin Antibody and a Cerebrovascular Accident. Response to Interferon Therapy', J Clin Gastroenterol, 24, 40-2.

40. Shilu Mathew, Muhammed Faheem, Sara M Ibrahim, et al. (2016). Hepatitis C Virus and Neurological Damage', World journal of hepatology, 8, 545.

41. JS McLaren, RH Stimson, ER McRorie, et al. (2001) The Diagnostic Value of Anti-Neutrophil Cytoplasmic Antibody Testing in a Routine Clinical Setting', Qjm, 94, 615-21.

42. S. Monaco, S. Ferrari, A. Gajofatto, et al. (2012) Hcv-Related Nervous System Disorders', Clin Dev Immunol, 2012, 236148.

43. Antonio Muscari, Giovanni M Puddu, Andrea Cenni, et al. (2009) Mean Platelet Volume (Mpv) Increase During Acute Non-Lacunar Ischemic Strokes', Thrombosis research, 123, 587-91.

44. Mohammed A Nafeh, Ahmed Medhat, Magda Shehata, et al. (2000) Hepatitis $C$ in a Community in Upper Egypt: I. Cross-Sectional Survey', The American journal of tropical medicine and hygiene, 63, 236-41.

45. Laura Origgi, Miriam Grasso, Massimo Vanoll, et al. (1998) Central Nervous System Involvement in Patients with Hcv-Related Cryoglobulinemia. The American journal of the medical sciences, 315, 20810.

46. G. W. Petty, J. Duffy, and J. Houston. (1996) Cerebral Ischemia in Patients with Hepatitis C Virus Infection and Mixed Cryoglobulinemia', Mayo Clinic Proceedings, 71, 671-78.

47. George W Petty, Joseph Dtjffy, and John Huston III. (1996) Cerebral Ischemia in Patients with Hepatitis C Virus Infection and Mixed Cryoglobulinemia', in Mayo Clinic Proceedings (Elsevier, 1996), pp. 671-78.

48. M Poljacki, Zorica Gajinov, Milana Ivkov, et al. (2000)Skin Diseases and Hepatitis Virus C Infection', Medicinski pregled, 53, 141-45.

49. Manuel Ramos-Casals, Veronique Loustaud-Ratti, Salvatore De Vita, et al. (2005) 'Sjögren Syndrome Associated with Hepatitis C Virus: A Multicenter Analysis of 137 Cases', Medicine, 84, 81-89.

50. Manuel Ramos-Casals, Angel Robles, Pilar BritoZerón, et al. (2006) Life-Threatening Cryoglobulinemia: Clinical and Immunological Characterization of 29 Cases', in Seminars in arthritis and rheumatism (Elsevier, 2006), pp. 189-96.

51. D. Sansonno, and F. Dammacco. (2005) Hepatitis C Virus, Cryoglobulinaemia, and Vasculitis: Immune Complex Relations', Lancet Infect Dis, 5, 227-36.

52. S. Sharaf-Eldeen, K. Salama, S. Eldemerdash, et al. (2007) Hepatitis B and C Viruses in Egyptian 
Children with Malignancy', Journal of Medical Sciences(Faisalabad), 7, 1003-08.

53. G. Stoll, and M. Bendszus. (2006) Inflammation and Atherosclerosis: Novel Insights into Plaque Formation and Destabilization', Stroke, 37, 1923-32.

54. G. Sulter, C. Steen, and J. De Keyser. (1999) Use of the Barthel Index and Modified Rankin Scale in Acute Stroke Trials', Stroke, 30, 1538-41.

55. Salvatore Sutti, Matteo Vidali, Cristina Mombello, et al. (2010) Breaking Self-Tolerance toward Cytochrome P4502e1 (Cyp2e1) in Chronic Hepatitis C: Possible Role for Molecular Mimicry', Journal of Hepatology, 53, 431-38.

56. Maria Tampaki, (2014) 'Extrahepatic Immune Related Manifestations in Chronic Hepatitis C Virus Infection', World Journal of Gastroenterology, 20, 12372.

57. G. Teasdale, and B. Jennett. (1974) Assessment of Coma and Impaired Consciousness. A Practical Scale', Lancet, 2, 81-4.

58. Stroke Unit Trialists'Collaboration, 'Organised Inpatient (Stroke Unit) Care for Stroke', Cochrane database syst rev, 4 (2007).

59. M. Uyttenboogaart, R. E. Stewart, P. C. Vroomen, et al. (2005) Optimizing Cutoff Scores for the Barthel Index and the Modified Rankin Scale for Defining Outcome in Acute Stroke Trials', Stroke, 36, 1984-7.

60. Nada Vasiljevic, and Ljiljana Markovic. (2005) Gene Similarity between Hepatitis C Virus and Human Proteins: A Blood Transfusion Problem', Medicinski pregled, 58, 582-86.
61. H. Völzke, C. Schwahn, B. Wolff, et al. (2004) Hepatitis $B$ and $C$ Virus Infection and the Risk of Atherosclerosis in a General Population', Atherosclerosis, 174, 99-103.

62. R. A. Watts, J. Mooney, J. Skinner, et al. (2012) The Contrasting Epidemiology of Granulomatosis with Polyangiitis (Wegener's) and Microscopic Polyangiitis', Rheumatology, 51, 926-31.

63. Kumi Yanagiha, Kazuhiro Ishii, Tomoyuki Ueno, et al. (2017) Medial Medullary Infarction Caused by Antineutrophil Cytoplasmic Antibody-Related Vasculitis: Case Report and Review of the Literature', Medicine, 96.

64. Z. M. Younossi, M. Stepanova, F. Nader, et al. (2013) Associations of Chronic Hepatitis C with Metabolic and Cardiac Outcomes', Aliment Pharmacol Ther, 37, 647-52.

65. R. Zampino, A. Marrone, L. Restivo, et al. (2013), Chronic Hcv Infection and Inflammation: Clinical Impact on Hepatic and Extra-Hepatic Manifestations', World J Hepatol, 5, 528-40.

66. F. Zancada Díaz de Entre-Sotos, M. T. Pérez-Aloe Mejías, and J. F. Pérez-Tovar. (2004) Stroke and Limb Ischaemia in Hepatitis C Virusrelated Cryoglobulinaemia', Irish Journal of Medical Science, 173, 57-57.

67. Claudia O Zein, Cynthia Levy, Ananda Basu, et al. (2005) Chronic Hepatitis C and Type li Diabetes Mellitus: A Prospective Cross-Sectional Study', The American journal of gastroenterology, 100, 48. 